\title{
CONTENIDO DE PLOMO SANGUÍNEO Y COMPOSICIÓN CORPORAL EN MUJERES JÓVENES EXPUESTAS A HUMO DE MADERA EN LA CIUDAD DE TEMUCO
}

\section{BLOOD LEAD LEVELS AND BODY COMPOSITION IN YOUNG WOMEN EXPOSED TO WOOD SMOKE IN THE CITY OF TEMUCO}

\author{
Rachel Pacheco A. (1), Camila Bay C. (1), Yareni Gutiérrez G. (2), Gabriela Salazar R.(3), \\ Luis Muñoz A. (4), Miguel Llanos S. (2), Ana María Ronco M. (2) \\ (1) Departamento de Ingeniería Química y Biotecnología, \\ Facultad de Ciencias Físicas y Matemáticas, U de Chile. Chile. \\ (2) Laboratorio de Nutrición y Regulación Metabólica. Santiago, Chile. \\ (3) Laboratorio de Metabolismo Energético e Isótopos Estables, \\ Instituto de Nutrición y Tecnología de los Alimentos (INTA), Universidad de Chile. Santiago, Chile. \\ (4) Sección de Metrología Química, Comisión Chilena de Energía Nuclear (CCHEN), La Reina, Santiago, Chile.
}

\begin{abstract}
One of the main concerns in the cities of developing countries is the coexistence of environmental pollution, malnutrition and overweight. The city of Temuco is highly polluted and the range of circulating lead of their inhabitants is unknown. The objective of this work was to study the potential association between blood lead concentration and body composition in young women $(n=45)$ living in the city of Temuco, which were exposed permanently to wood smoke. Blood lead concentration was analyzed by atomic absorption spectrometry with graphite furnace $(A A S-G F)$ and the body composition was determined by isotopic dilution (deuterium). All participants presented high percentage of fat mass (FM) and $66.7 \%$ of them had blood lead levels over the accepted international limits $(5 \mathrm{\mu g} / \mathrm{dl}$; EPA; USA), although lower than the Chilean normative (40 $\mu \mathrm{g} / \mathrm{dl})$, which were not correlated with the $\%$ of FM. The high percentage of overweight and obesity together with the lead blood levels found in this study constitute risk factors that may affect health and life quality of the participants in the medium term.
\end{abstract}

Key words: Lead, body composition, obesity, Temuco city.

Este trabajo fue recibido el 6 de Abril de 2011 y aceptado para ser publicado el 5 de Octubre de 2011.

\section{INTRODUCCIÓN}

En algunos países en desarrollo, la población está expuesta a factores ambientales adversos. El elevado nivel de contaminantes ambientales (metales tóxicos, material particulado) coexiste con una malnutrición poblacional, y los efectos que esta situación puede producir a mediano y largo plazo en la salud de la población expuesta se desconocen $(1,2)$.

El plomo $(\mathrm{Pb})$ es un metal encontrado naturalmente en rocas y suelos; también se utiliza en la fabricación de baterías, soldaduras (especialmente en componentes eléctricos y radiadores de autos), aleaciones de metales, plásticos, cerámica vidriada, entre otros. En ciertos países aún se comercializa pinturas y gasolina con $\mathrm{Pb}$. Una vez que es inhalado a través del material particulado fino $\mathrm{o}$ ingerido en forma soluble, este metal es absorbido, e incorporado a los eritrocitos y distribuido a múltiples tejidos, incluido el cerebro, riñones, médula ósea, y gónadas (3). Se ha reportado que concentraciones sanguíneas de $\mathrm{Pb}$, aún menores que $10 \mu \mathrm{g} / \mathrm{dl}(0.48 \mu \mathrm{mol} / \mathrm{l})$, se asocian con un aumento de riesgo cardiovascular, cáncer y mortalidad por todas las causas $(4,5)$.

$\mathrm{El} \mathrm{Pb}$ es capaz de atravesar la placenta y llegar al feto donde produce efectos deletéreos en el crecimiento 
y desarrollo fetal (6). Se ha observado recientemente una correlación negativa entre los niveles de $\mathrm{Pb}$ en la placenta humana y el peso de nacimiento $(7,8)$.

Debido al riesgo que puede tener para el feto en desarrollo que la madre esté expuesta a $\mathrm{Pb}$ durante el embarazo, las mujeres en edad reproductiva representan un grupo vulnerable de la población para las cuales el biomonitoreo de $\mathrm{Pb}$ puede ser importante, especialmente en ciudades contaminadas.

Temuco es la capital de la IX Región de la Araucanía y de la Provincia de Cautín, Chile, se ubica a 670 kilómetros al sur de Santiago y se caracteriza por poseer en su población una variedad étnica (9). Debido al crecimiento de su población y las actividades humanas que conlleva el desarrollo, la calidad del aire ha empeorado notablemente debido principalmente al uso masivo de combustión a leña tanto en el sector urbano como rural, con bajas orientaciones técnicas y ambientales (10). En consecuencia, la mayoría de la leña que se comercializa en Temuco, presenta mayores contenidos de humedad a los recomendados internacionalmente. Esto, combinado con malas condiciones de operación y tecnología de combustión deficiente, resultan en tasas superiores de emisión de material particulado fino comparado a los niveles de emisión internacionales (11). Adicionalmente, se ha reportado que la quema de leña libera al medio ambiente una cantidad de 0.3 a $9 \mathrm{mg}$ de $\mathrm{Pb}$ por cada 3 $\mathrm{Kg}$ de leña combustionada (12) las que provienen en un $74 \%$ de combustión residencial, un $18 \%$ de la industria y el comercio $6 \%$ y un $2 \%$ del transporte público y privado (13 - 15).

Hay pocos estudios en la literatura que relacionen niveles de metales tóxicos en fluidos biológicos con el estado nutricional; en ellos se ha reportado una asociación entre la exposición a Pb y deficiencia nutricional $(1,2)$. A niveles de exposición a $\mathrm{Pb}$ similares, los síntomas adversos en la población con déficit nutricional fueron mayores que en la población con un estado nutricional adecuado (16). Sin embargo, en el estudio mencionado, no se determinaron los niveles de $\mathrm{Pb}$ en fluidos biológicos, y además, no se evaluó el sobrepeso u obesidad en relación a la exposición al tóxico. El único estudio de este tipo realizado en Chile y reportado por nosotros en una población en Santiago, seleccionada de acuerdo al estado nutricional, no arrojó asociación entre los niveles de los elementos tóxicos estudiados (plomo y arsénico) y el porcentaje de masa corporal (17). En un estudio previo realizado en niños tampoco se encontró una asociación entre los niveles sanguíneos de $\mathrm{Pb}$ y masa corporal (18). En ratones, la exposición prenatal $\mathrm{a} \mathrm{Pb}$ indujo en las crías macho un aumento en el peso corporal a la edad de un año (19).
El objetivo del presente estudio fue determinar en una muestra de mujeres jóvenes expuestas a humo de leña, la concentración de $\mathrm{Pb}$ sanguíneo y su composición corporal para establecer una potencial asociación entre estas variables.

\section{SUJETOS Y MÉTODOS Sujetos participantes}

Los sujetos participantes en el estudio fueron reclutados en el consultorio de la ciudad de Temuco. Se invitó a participar a mujeres en edad reproductiva ( $\mathrm{n}=45 ; 25-35$ años) residentes en Temuco y que estuvieran en contacto permanente con combustión a leña (principalmente cocinas y chimeneas) al interior de sus hogares. Las mujeres que aceptaron participar fueron sometidas a distintos criterios de exclusión e inclusión (rango de edad, sin patologías crónicas ni agudas, no embarazadas, ni en período de lactancia). Este estudio fue aprobado por el Comité de Ética del Instituto de Nutrición y Tecnología de los Alimentos (INTA, Universidad de Chile). Todas las mujeres reclutadas fueron encuestadas para determinar su nivel socioeconómico y firmaron un consentimiento informado. Posteriormente, se les determinó el peso y la talla para evaluar su índice de masa corporal (IMC: peso $(\mathrm{kg}) / \mathrm{talla}\left(\mathrm{m}^{2}\right)$ de acuerdo al cual se clasificaron.

\section{Encuesta Socioeconómica}

El nivel socioeconómico fue evaluado a través de la escala propuesta en la encuesta de Graffar modificada (20). La encuesta consideró las siguientes variables: escolaridad, número de personas que conviven en el hogar, jefe de hogar, trabajo e ingresos mensuales del grupo familiar, previsión, casa propia, calidad de la construcción en la que reside, infraestructura y servicios básicos, agua potable, baños, bienes: TV, cocina, auto, computador. A cada variable se le asignó un puntaje, el que ponderado entregó un puntaje final que permitió clasificarlos en nivel socioeconómico alto- medio alto - medio - medio bajo - bajo.

\section{Método de dilución isotópica para determinar composición corporal}

La composición corporal fue determinada por el método de dilución isotópica (deuterio), basado en el modelo de composición corporal de dos compartimentos (masa grasa: MG y masa libre de grasa; MLG) (21). El agua se encuentra exclusivamente en la MLG, por lo tanto, a partir de la medición del agua corporal total (ACT) y asumiendo un coeficiente de hidratación en los adultos del $73.2 \%$, se puede estimar la MLG (MLG= ACT/0.732) y luego por diferencia con el peso corporal 
la $\mathrm{MG}$ (MG= peso - MLG) (21).

El principio de este método es la dilución. El deuterio en concentración $(\mathrm{C})$ y volumen $(\mathrm{V})$ conocidos $(\mathrm{C} 1$ y V1), se diluye en el organismo con concentración y volumen desconocidos (C2 y V2). La nueva concentración (C2) es medida en fluidos biológicos (saliva) por un espectrómetro de masas y después el V2 (ACT) puede ser calculado con la ecuación 1.

$$
\mathrm{V} 2=\frac{\mathrm{V} 1 * \mathrm{C} 1}{\mathrm{C} 2}
$$

Después de $12 \mathrm{~h}$ de ayuno se tomó una muestra basal de saliva de $4 \mathrm{ml}$ de cada participante, inmediatamente después cada una de ellas ingirió primero una dosis de deuterio conocida ( $3 \mathrm{~g}$ ) y luego $40 \mathrm{ml}$ de agua de enjuague; 3 horas después (tiempo de estabilización del deuterio), una segunda muestra de saliva fue recolectada (muestra post dosis) (22). Las muestras fueron etiquetadas y congeladas hasta que se analizaron en un espectrómetro de masas (Europa Scientific, Hydra 2020). Una vez obtenido el porcentaje de la masa grasa de las participantes, se clasificaron en normal $(<31 \% \mathrm{MG})$, con sobrepeso (31-33\% MG) y obesas (>33\% MG) (17).

\section{Determinaciones de plomo en sangre}

$\mathrm{El} \mathrm{Pb}$ se determinó en una muestra de sangre tomada por venopunción ( $3 \mathrm{ml}$ ), después de $12 \mathrm{~h}$ de ayuno, la que fue recolectada y guardada en tubos vacutainers libres de elementos traza (tapa azul). Se congelaron a $-20{ }^{\circ} \mathrm{C}$ para determinación posterior de $\mathrm{Pb}$ por espectrofotometría de absorción atómica con horno de grafito (AAS-GF, AAS 5 E-A, Carl Zeiss), previa digestión con $\mathrm{HNO}_{3}-\mathrm{H}_{2} \mathrm{O}_{2}$. El límite de detección (LOD) fue de $1.99 \mu \mathrm{g} / \mathrm{dl}$ y se utilizó sangre de bovino seca congelada como material de referencia (GBW No. 09139, $111 \mu \mathrm{g} \mathrm{Pb/l).}$

A los valores reportados bajo $2 \mu \mathrm{g} / \mathrm{dl}$ se les asignó el valor de $1 \mu \mathrm{g} / \mathrm{dl}$ para el análisis estadístico (16).

\section{Análisis bioquímicos}

A las pacientes se les realizó un perfil lipídico: CT: colesterol total, HDL-colesterol; LDL-colesterol, triglicéridos y además, se determinó la concentración plasmática de Zn-protoporfirina (Zpp, marcador de exposición crónica a $\mathrm{Pb}$ ).

\section{Análisis estadístico}

El análisis de datos se realizó usando el programa estadístico SPSS versión 11.5 para Windows (SPSS Inc., Chicago, USA). Se evaluó la consistencia de los datos y la presencia de valores extraños (outlayers) seguido de la aplicación del test de Shapiro Wilk para evaluar la normalidad de las variables. Se aplicó estadística des- criptiva para todas las variables. Para las variables que tuvieron distribución normal se calculó el promedio y desviaciones estándar (DE); en caso contrario los resultados se expresaron como medianas y rango intercuartil (RI). Para evaluar las diferencias de todas las variables entre las categorías de IMC y composición corporal se aplicó ANOVA cuando se cumplía con el supuesto de normalidad y homogeneidad de varianza; Kruskal Wallis cuando las variables no tenían distribución normal ó Chi cuadrado para variables categóricas.

Para evaluar si existían diferencias entre los niveles de $\mathrm{Pb}$ y el \% MG se aplicó la prueba no paramétrica de Kruskal Wallis debido a que la variable dependiente $(\mathrm{Pb})$ no tuvo una distribución normal.

\section{RESULTADOS}

En la tabla 1 se describe las características de las mujeres participantes en el estudio. Al clasificar a las participantes por IMC, 5 tuvieron peso normal (IMC > $\left.19 \leq 24.9 \mathrm{Kg} / \mathrm{m}^{2}\right), 20$ fueron clasificadas con sobrepeso $\left(\mathrm{IMC}>25<30 \mathrm{Kg} / \mathrm{m}^{2}\right.$ ) y 20 fueron obesas (IMC $>30.5$ $\mathrm{Kg} / \mathrm{m}^{2}$ ). Tanto la edad como la talla no fueron factores determinantes del estado nutricional. El \% de MG calculado por el método de dilución isotópica fue de $36.0 \%$, $40.6 \%$ y $45.5 \%$ para las mujeres de peso normal, con sobrepeso y obesas respectivamente, clasificadas previamente de acuerdo a su IMC. Dado que en base al \% de MG los sujetos se clasifican como normales: \% MG $<30 \%$, con sobrepeso: $\%$ de $\mathrm{MG}>31<33 \%$ y obesas: $\%$ de $\mathrm{MG}>33 \%$, una re-clasificación de las participantes de acuerdo a su $\%$ de MG, define a todas las participantes como obesas (>33\% de MG). El 62.2\% de las participantes se clasificó en el grupo socioeconómico medio, y no se observaron diferencias en el estado socioeconómico entre las participantes con diferente IMC (tabla 2). No se observaron diferencias entre los grupos en distintos parámetros bioquímicos analizados (tabla 3). De acuerdo al IMC de las participantes (tabla 4), los valores de las medianas de los niveles de $\mathrm{Pb}$ en sangre fueron 5.5, 6.6 y $8.25 \mu \mathrm{g} / \mathrm{dl}$ para las mujeres normales, con sobrepeso y obesas respectivamente. $\mathrm{Si}$ bien los niveles de $\mathrm{Pb}$ en sangre fueron mayores en las mujeres con sobrepeso y en mujeres con obesidad, esta diferencia no fue significativa. Al subdividir a las participantes en rangos, de acuerdo a las concentraciones de $\mathrm{Pb}$ sanguíneos (tabla 5), se observó que $17.8 \%$ de las mujeres tenía niveles de $\mathrm{Pb}$ sanguíneos inferiores a $2 \mathrm{mg} / \mathrm{dl}$, un $48.9 \%$ tiene niveles $>2<10 \mu \mathrm{g} / \mathrm{dl}$, mientras que un $33.3 \%$ presenta niveles superiores a $10 \mu \mathrm{g} / \mathrm{dl}$. Además, se registraron dos mujeres con valores superiores a los $100 \mu \mathrm{g} / \mathrm{dl}$ pertenecientes al grupo de obesas. No se encontró una correlación entre los niveles sanguíneos de $\mathrm{Pb}$ y el \% de MG. 


\section{TABLA 1}

Características antropométricas de las mujeres participantes.

$\begin{array}{lcccccc}\text { Variable } & \text { Estadística } & \text { IMC } & \text { Total } & \text { P } \\ & & \text { Normopeso } & \text { Sobrepeso } & \text { Obesidad } & & \\ \text { Edad (años) } & \text { Mediana } & 34.0 & 32.5 & 30 & 30.0 & \\ & (\mathrm{RI}) & (28.5-34.5) & (28.25-34.0) & (28.0-31.75) & (28.0-34.0) & 0.220 \\ \text { Peso }(\mathrm{Kg}) & \text { Mediana } & 56.2 & 67.7 & 84.8 & 71.8 & \\ & (\mathrm{RI}) & (53.1-57.5) & (64.5-71.4) & (77.2-91.0) & (65.7-80.5) & 0.000 \\ \text { Talla }(\mathrm{cm}) & \text { Promedio } & 154.4 & 156.8 & 155.8 & 1.56 & \\ & (\mathrm{DE}) & (0.03) & (0.64) & (0.03) & (0.48) & 0.580 \\ \text { IMC }\left(\mathrm{Kg} / \mathrm{m}^{2}\right) & \text { Mediana } & 23.5 & 27.1 & 35.5 & 28.6 & \\ & (\mathrm{RI}) & (22.3-24.2) & (26.1-28.5) & (31.7-38.2) & (26.5-33.6) & 0.000 \\ \text { Agua }(\%) & \text { Promedio } & 46.9 & 43.6 & 39.9 & 42.3 & \\ & (\mathrm{DE}) & (1.20) & (1.90) & (3.25) & (3.44) & 0.000 \\ \text { Masa grasa }(\%) & \text { Promedio } & 36.0 & 40.6 & 45.5 & 42.2 & \\ & (\mathrm{DE}) & (1.64) & (2.60) & (4.43) & (4.69) & 0.000\end{array}$

TABLA 2

Nivel socioeconómico de las mujeres participantes.

\begin{tabular}{|lcccc|}
\hline $\begin{array}{l}\text { Situación } \\
\text { Socioeconómica }\end{array}$ & $\begin{array}{c}\text { Número } \\
\text { de mujeres }\end{array}$ & Porcentaje \% & Promedio IMC & $\begin{array}{c}\text { Desviación } \\
\text { estándar IMC }\end{array}$ \\
\hline Bajo & 2 & 4.4 & 25.1 & 1.48 \\
Medio Bajo & 8 & 17.8 & 36.9 & 4.73 \\
Medio & 28 & 62.2 & 29.4 & 4.22 \\
Medio Alto & 7 & 15.6 & 29.2 & 6.57 \\
\hline
\end{tabular}

\section{TABLA 3}

Parámetros bioquímicos sanguíneos

\begin{tabular}{lccc}
\hline Medición & Normal & Sobrepeso & Obesa \\
\hline Zpp (mg/dl GR) & $53 \pm 9$ & $56 \pm 17$ & $64 \pm 38$ \\
Colesterol total (mg/dl) & $178 \pm 39$ & $178 \pm 36$ & $191 \pm 32$ \\
HDL-C (mg/dl) & $54 \pm 1$ & $50 \pm 8.5$ & $45.6 \pm 7$ \\
LDL-C (mg/dl) & $109 \pm 32$ & $101 \pm 31$ & $122 \pm 31$ \\
TG (mg/dl) & $75 \pm 38$ & $106 \pm 61$ & $145 \pm 81$ \\
\hline Zpp: zinc protoporfirina; GR: glóbulos rojos; HDL-C: colesterol unido a lipoproteína de alta densidad; \\
LDL-C: colesterol unido a lipoproteína de baja densidad; TG: triglicéridos. ANOVA.
\end{tabular}




\section{DISCUSIÓN}

Este estudio reveló que los niveles de $\mathrm{Pb}$ sanguíneos en una pequeña muestra de mujeres jóvenes residentes en la ciudad de Temuco (mediana: $>5,5 \mu \mathrm{g} / \mathrm{dl}$ ) están sobre los valores reportados en un estudio en el mismo grupo etario realizado en Santiago (17) y otro estudio realizado en los EEUU, en el cual en una población representativa de más de 13.000 adultos se encontró un promedio geométrico de $\mathrm{Pb}$ sanguíneo de 2,58 $\mu \mathrm{g} / \mathrm{dl}$ (4).

El segundo hallazgo que llama la atención de este estudio es el gran porcentaje de obesidad en mujeres del grupo etario estudiado. Al clasificarlas según su IMC, sólo 5 de ellas, de un total de 45 fueron catalogadas como "normales", sin embargo al analizar su \% de MG, éstas se re-clasifican como obesas. En consecuencia, las mujeres previamente clasificadas como normales, por su IMC, de acuerdo al \% de MG resultan obesas por lo que ninguna mujer participante en este estudio puede ser considerada como normal desde la perspectiva de su composición corporal. Este resultado es relevante desde el punto de vista nutricional y pone una voz de alerta al elevado porcentaje de mujeres con sobrepeso en este grupo etario. Se desconocen las razones, principalmente debido a que no se hicieron encuestas sobre hábitos alimenticios.

Al revisar antecedentes del Ministerio de Salud sobre hábitos de la dieta de mujeres de la IX Región $(23,24)$, se observa que el año 2006 sólo un 75.4\% declaró tomar desayuno todos los días y un $50.9 \%$ dice comer verduras todos los días. Además, en una encuesta a mujeres entre 20 a 44 años, sólo un $7.3 \%$ respondió afirmativamente practicar algún deporte o actividad física en el último mes, fuera de su horario de trabajo, durante 30 minutos o más 3 o más veces por semana. El $58.0 \%$ de las mujeres entrevistadas de todas las edades dijeron no estar haciendo nada actualmente para mantener controlado su peso, es decir, para no subir de peso (23). En 2001, mujeres entre 20 y 44 años afirmaron en

\section{TABLA 4}

\section{Niveles de plomo sanguíneos según el Índice de Masa Corporal}

\begin{tabular}{|lcccccc} 
& & \multicolumn{3}{c}{ IMC } & & \\
Variable & Estadístico & Normopeso & Sobrepeso & Obesidad & Total & P \\
\hline $\mathrm{Pb}$ en sangre $(\mu \mathrm{g} / \mathrm{dl})$ & Mediana & 5.5 & 6.6 & 8.25 & 6.7 & \\
& $(\mathrm{RI})$ & $(0.4-8.8)$ & $(4.7-11.0)$ & $(0.3-17.4)$ & $(3.0-14.8)$ & 0.575 \\
\hline Test de Kruskal-Wallis. & & & & & &
\end{tabular}

\section{TABLA 5}

Niveles de plomo sanguíneos por estado nutricional

\begin{tabular}{lccccc} 
& & \multicolumn{2}{c}{$\mathbf{P b}(\boldsymbol{\mu} \mathbf{g} / \mathbf{d l})$} & & \\
IMC & $<\mathbf{2}$ & $\mathbf{2 - 1 0}$ & $\mathbf{1 0 - 4 0}$ & $\mathbf{4 0}$ & Total \\
Normopeso & 2 & 2 & 1 & 0 & 5 \\
Sobrepeso & $40.0 \%$ & $40.0 \%$ & $20.0 \%$ & $0 \%$ & $100 \%$ \\
& 2 & 12 & 6 & 0 & 20 \\
Obesa & $10.0 \%$ & $60.0 \%$ & $30.0 \%$ & $0 \%$ & $100.0 \%$ \\
& 6 & 6 & 6 & 2 & 20 \\
Total & $30.0 \%$ & $30.0 \%$ & $30.0 \%$ & $10.0 \%$ & $100.0 \%$ \\
& 10 & 20 & 13 & 2 & 45 \\
$\mathrm{X}^{2}=7.304, \mathrm{p}=0.294$ & $22.2 \%$ & $44.4 \%$ & $28.9 \%$ & $4.4 \%$ & $100.0 \%$
\end{tabular}


un $31.9 \%$ comer carne de vacuno 2 ó 3 veces por semana, un $10.6 \%$ carne de cerdo, cordero, cecinas o interiores 2 ó 3 veces por semana y un $21.3 \%$ dice que rara vez toma leche (24).

Es importante reconocer que sólo el $4.4 \%$ de las mujeres participantes pertenecía a un estrato socioeconómico bajo por lo que no se puede argüir un desconocimiento de los beneficios del ejercicio como herramienta para controlar el peso corporal. La práctica de actividad física está condicionada por el clima (lluvia, mal tiempo, temporales, entre otros) y el hecho de que no siempre se dispone de espacios físicos adecuados ni tampoco existe la voluntad para realizarlos. Se puede deducir que esta situación promueve la realización de convivencias sociales, casi siempre en torno a la comida, en general frituras o productos hechos con papa o harina, ya sea para comercializarlas como beneficio o simplemente como arte de compartir (24). Este hábito ha sido considerado un argumento para postular que los niveles elevados de colesterol pueden llegar a constituir un problema de salud en la zona sur del país (25), debido a que el clima lluvioso y frío ayuda a aumentar el consumo de alimentos con elevado contenido graso. Sin embargo, no se encontraron parámetros lipídicos alterados que indiquen un riesgo de salud por el consumo de alimentos de alto contenido graso o calórico. Tampoco se observaron niveles elevados de zinc-protoporfirina (Zpp), lo que indica ausencia de exposición crónica a $\mathrm{Pb}$ en las participantes del estudio.

Es relevante y preocupante a la vez que un $66.7 \%$ de las mujeres participantes tengan una concentración de $\mathrm{Pb}$ sanguíneo por sobre los valores aceptados internacionalmente ( $5 \mu \mathrm{g} / \mathrm{dl}$; EPA; USA); estando el $33.3 \%$ de ellas en severo riesgo de salud al registrar concentraciones de $\mathrm{Pb}$ mayores a $10 \mu \mathrm{g} / \mathrm{dl}$. Aunque este estudio no nos permite determinar las fuentes precisas de exposición a $\mathrm{Pb}$, es altamente probable que provenga del creciente grado de contaminación de la ciudad de Temuco debido al alto uso de chimeneas y estufas a leña en los hogares. Las concentraciones de $\mathrm{Pb}$ encontradas en este estudio son mayores que las reportadas en un estudio realizado en USA, donde sólo el $1 \%$ de las muestras registró niveles $>10 \mu \mathrm{g} / \mathrm{dl}$ (4). Los niveles sanguíneos de $\mathrm{Pb}$ constituyen un indicador de exposición reciente, lo que significa que las mujeres participantes están, en sus actividades diarias, expuestas a bajas concentraciones de $\mathrm{Pb}$. Dado que la normativa chilena es mucho menos exigente en relación a las concentraciones máximas de $\mathrm{Pb}$ aceptadas en la sangre (40 $\mu \mathrm{g} / \mathrm{dl}$; DS. 594/99), solo un $4.4 \%$ está sobre los valores aceptados. Estos valores son considerados tóxicos y no se tiene información sobre la fuente directa de exposición.
Estudios recientes señalan que concentraciones de $\mathrm{Pb}$ sanguíneas muy bajas, incluso del orden de $1 \mu \mathrm{g} / \mathrm{dl}$ y muy inferior a la normativa chilena, está asociada a efectos adversos en la salud y a un aumento de la mortalidad por todas las causas. En niños en edad escolar, la observación que niveles sanguíneos de $\mathrm{Pb}$ muy bajos están asociados a trastornos cognitivos y de aprendizaje $(26,27)$, confirma que a niveles mucho más bajos que los aceptados inducen efectos adversos a la salud y con consecuencias a largo plazo.

En estudios con niños desnutridos, la absorción de $\mathrm{Pb}$ fue mayor que la de niños con un buen estado nutricional frente a un nivel de exposición similar (28). Esta situación no es aplicable a este estudio, ya que ninguna de las mujeres presentó algún grado de desnutrición.

Se ha reportado que el humo de leña puede causar efectos respiratorios adversos (29). El tamaño promedio de las partículas contenidas en él $(<1 \mu \mathrm{m})$ puede viajar muy profundo hasta el tracto respiratorio inferior, por lo que algunas especies químicas reactivas presentes en el humo llegan hasta los tejidos respiratorios, transformándose en una verdadera amenaza. Pueden llegar hasta los alvéolos, irritantes como fenoles, aldehídos y quinonas, como también óxidos de nitrógeno y sulfuro, que pueden contribuir tanto a agravar como hacer crónico algún problema de salud $(29,30)$.

Se ha reportado en Nueva Guinea, India y Nepal un aumento en los síntomas respiratorios, función pulmonar disminuida y gran aumento en la prevalencia de bronquitis crónica (30). En México, 30 personas no fumadoras, que vivían en el campo lejos de contaminación urbana, expuestas a humo producto de la quema de madera para cocinar, presentaron radiografías de pecho anormales evidenciando hipertensión arterial pulmonar. Se sugirió que sufrían de "wood-smoke-inhalation-associated lung disease (WSIALD)" que en español significaría enfermedad a los pulmones asociada a la inhalación de humo de leña (31). Por lo tanto, es importante evitar la inhalación de humo de leña.

En resumen, este estudio realizado en una muestra aleatoria de mujeres jóvenes residentes en Temuco, nos muestra dos situaciones alarmantes: que sus niveles sanguíneos de Pb están por sobre los niveles "normales" en ciudades comparables de desarrollo y polución y que, el $100 \%$ registró un elevado \% de MG. No se encontró una relación entre el IMC o porcentaje de MG y los niveles $\mathrm{de} \mathrm{Pb}$ en sangre.

Finalmente, se recomienda a los organismos competentes de salud realizar modificaciones a la normativa chilena para reducir los niveles aceptados de $\mathrm{Pb}$ en sangre desde $40 \mu \mathrm{g} / \mathrm{dl}$ a valores similares a la normativa internacional $(5 \mu \mathrm{g} / \mathrm{dl})$. 


\section{RESUMEN}

Uno de los problemas más frecuentes en las ciudades de países en desarrollo es la coexistencia de polución ambiental, malnutrición y sobrepeso. La ciudad de Temuco posee una elevada contaminación ambiental y se desconoce el rango de las concentraciones de plomo circulante en la población. El objetivo de este trabajo fue estudiar la concentración de plomo sanguíneo en mujeres temuquenses expuestas a humo de leña doméstico en forma continua $(n=45)$, y su posible asociación con la composición corporal. La concentración sanguínea de plomo fue analizada por espectrofotometría de absorción atómica con horno de grafito (AAS-GF), y la composición corporal se determinó por el método de dilución isotópica (deuterio). Todas las mujeres participantes registraron elevados porcentajes de masa grasa (MG) y un $66.7 \%$ de ellas registró concentraciones de plomo sanguíneo sobre los niveles aceptados internacionalmente (5 $\mu \mathrm{g} / \mathrm{dl}$; EPA; USA), aunque bajo la normativa chilena (40 $\mu \mathrm{g} / \mathrm{dl})$, los que no se correlacionaron con el \% de MG. El elevado porcentaje de sobrepeso y obesidad junto a los niveles de plomo observados en este estudio constituyen factores de riesgo que afectarán en un mediano plazo la salud y calidad de vida de las participantes.

Palabras clave: plomo; composición corporal; obesidad; Temuco.

\section{Dirigir la correspondencia a:}

Profesora

Ana María Ronco M.

INTA - Universidad de Chile

El Líbano 5524, Macul. Santiago, Chile

Fono: 9781430

Fax: 2214030

E-mail:amronco@inta.uchile.cl

Agradecimientos: Los autores agradecen el financiamiento de la Organización Internacional de Energía Atómica (IAEA, RC No 13245 RBF) y la colaboración de la nutricionista Marcia Velásquez, MSc. y del nutricionista Sr. Rodrigo H. Torres por su participación en la toma de muestras de las participantes.

Financiamiento: Organización Internacional de Energía Atómica (IAEA), RC No 13245 RBF.

Ambas autoras Rachel Pacheco y Camila Baez contribuyeron de igual forma al estudio.

\section{BIBLIOGRAFÍA}

1. Milton A, Hasan Z, Shahidullah S, Sharmin S. Association between nutritional status and arsenicosis due to chronic arsenic exposure in Bangladesh. Int J Environ Health Res 2004; 4:99-108.
2. Islam L, Nabi A, Rahman M, Khan M. Association of clinical complications with nutritional status and the prevalence of leukopenia among arsenic patients in Bangladesh. Int J Environ Res Public Health 2004; 1:74-82.

3. Third National Report on Human Exposure to Environmental Chemicals.

4. Menke A, Muntner P, Batuman V. Blood lead below $0.48 \mathrm{micromol} / \mathrm{L}$ (10 microg/dL) and mortality among US adults. Circulation 2006; 114:1388-94.

5. Lustberg M, Silbergeld E. Blood lead levels and mortality. Arch Intern Med 2002; 62:2443-9.

6. Lee MG, Chun OK, Song WO. Determinants of the blood lead level of US women of reproductive age. J Am Coll Nutr 2005; 24:1-9.

7. Llanos MN, Ronco AM. Fetal growth restriction is related to placental levels of cadmium, lead and arsenic but not with antioxidant activities. Reprod Toxicol 2009; 188:186-91.

8. Ronco AM, Arguello G, Munoz L. Metals content in placentas from moderate cigarette consumers: correlation with newborn birth weight. Biometals 2005 ; $18: 233-41$.

9. Temwen, nuestraciudad [en línea] http://www.temucochile.com/indextco.htm [consulta: 16 mayo 2010]

10. Bustos S. Comienzan episodios críticos de contaminación en Temuco [en línea]_Emol, El_Mercurio on line 25 de abril, 2010 http./www emoll $\mathrm{com} /$ noticias/nacional/detalle/detallenoticias. asp?idnoticia=409822 [consulta: 25 mayo 2010]

11. Escobar J, Jorquera H, Bordones JC, Pfeng C, Palacios R, Tardón MJ, Daroch P, Miranda M, Oyaneder E. Actualización del inventario de emisiones atmosférica en las comunas de Temuco y Padre Las Casas. Informe técnico celebrado entre Comisión Nacional del Medio Ambiente Región de la Aramcanía y DICTUCS A 2008 [en línea] http. www.sinia.cl/1292/articles-46042_recurso_1.pdf [consulta: 8 junio 2010]

12. Larson TV, Koenig JQ. Wood smoke: emissions and non-cancer respiratory effects. Ann Rev Public Health 1994;15:133-56.

13. Otero L, Lobos M, Vera A, Kausel T. Estudio: Generación de antecedentes para la implementación de un Sistema Nacional de Certificación de Leña. Informe final corregido Propuesta de un Sistema Nacional de Certificación de Leña. Desarrollado para CONAMA Región de La Araucanía 2004 [en línea] http./www.sinia.cl/1292/articles-46038_recurso_1.pdf [consulta: 10 junio 2010]

14. Universidad de Concepción. Priorización de medidas de reducción de emisiones por uso residencial de 
leña para la gestión de la calidad del aire en Temuco y Padre Las Casas. Contrato $\mathrm{N}^{\circ} 14-22-008 / 01$ Informe Final 2002 [en_línea] http./Www.sinia. cl/1292/articles-28474_recurso_1.pdf [consulta: 10 junio 2010]

15. Comisión Nacional del Medio Ambiente, CONAMA IX Región. Estudio: Percepción y disposición ciudadana de la gestión en calidad de aire realizada en Temuco y Padre Las Casas 2007 [en línea] s http://www.sinia.cl/1292/articles-46041_recurso_1. pdtp [consulta: 13 junio 2010]

16. Mahaffey K Environmental lead toxicity: nutrition as a component of intervention. Environ Health Perspect 1990; 89:75-8.

17. Ronco AM, Gutierrez Y, Gras N, Muñoz L, Salazar $\mathrm{G}$, Llanos MN. Lead and arsenic levels in women with different body mass composition. Biol Trace Elem Res 2010; 136: 269-78.

18. Huzior-Bałajewicz A, Pietrzyk J J, Schlegel-Zawadzka M, Piatkowska E, Zachwieja Z. The influence of lead and cadmium environmental pollution on anthropometric health factors in children. Przegl Lek. 2001; 58:315-24.

19. Leasure JL, Giddabasappa A, Chaney S, Johnson JE, Pothakos K, Lau YS, Fox DA. Low-level human equivalent gestational lead exposure produces sex-specific motor and coordination abnormalities and late-onset obesity in year-old mice. Environ Health Perspect 2008; 116:355-61.

20. Alvarez M, Ivanovic D. Escala para medición del nivel socioeconómico en el área de la salud. Rev Méd Chile 1985;113: 243-9.

21. Ellis KJ. Selected body composition methods can be used in field studies. J Nutr 2001; 131:1589S-95S.

22. Eckhardt CL, Adair LS, Caballero B, Avila J, Kon IY, Wang J, Popkin BM . Estimating body fat from anthropometry and isotopic dilution: a four - country comparison, Obesity Res 2003; 11:1553-62.

23. Departamento de Epidemiología-Ministerio de Salud. II Encuesta de calidad de vida y salud, Chile 2006. Resultados regionales y por zona rural y ur- bana. 2006 [en línea] <http://epi.minsal.cl/epi/html/ sdesalud/calidaddevida2006/index.htm $>$ [consulta: 10 agosto 2010]

24. Departamento de Epidemiología \& Departamento de Promoción de la Salud-Ministerio de Salud. I Encuesta de calidad de vida y salud, Chile 2001. Informe regional de factores de riesgo. Novena Región. 2001 [en línea] <http://epi.minsal.cl/epi/ html/sdesalud/cdevid/cdvregf/final9region.pdf $>$ [consulta: 9 agosto 2010]

25. Cuyul C, Agüero E. Altos niveles de colesterol en el sur de Chile QQué hacer? 2006 [en línea] http.//medicina wach cl/saludpublica/diplomado entenido/trahaios/1/0sorno\% $202006 / 4$ tas $\% 20$ Niveles\%20de\%20Colesterol\%20en\%2gel\%20 Sur\%20de\%20Chile\%20Que\%20hacer.pdf [consulta: 12 agosto 2010]

26. Surkan PM, Zhang A, Trachtenberg F, Daniel DB, McKinlay S, Bellinger DC. Neuropsychological function in children with blood lead levels $<10 \mathrm{mg} /$ dL. NeuroToxicology 2007; 28: 1170-7.

27. Jusko TA, Henderson CR, Lanphear BP, CorySlechta DA, Parsons PJ, Canfield RL. Blood Lead Concentrations $<10 \mu \mathrm{g} / \mathrm{dL}$ and Child Intelligence at 6 Years of Age. Environ Health Perspect 2008; 116:243-8.

28. Schwartz J, Angle C y Pitcher H. Relationship between Childhood Blood Lead Levels and Stature. Pediatrics 1986; 77: 281-8.

29. Ammann H. Health implications of Wood smoke. International Congress on Residential Wood Energy; Conferences and Institutes, Reno. 1986. WSU WWREC pp 331-48, 1986.

30. Reid H, Smith K, Sherchand B. Indoor smoke exposures from traditional and improved cookstoves: Comparisons among rural Nepali women. Mountain Res Devel 1986;6: 293-304.

31. Sandoval J, Salas J, Martinez-Garcia M, Gomez A, Martinez C, et al. Pulmonary arterial hypertension and cor pulmonale associated with chronic domestic woodsmoke inhalation. 1992. Chest 103: 12-20. 